UT-842

YITP-99-21

\title{
Grand-Unification Scale Generation through the Anomalous U(1) Breaking
}

\author{
Izawa K.-I. ${ }^{1,2}$, Kiichi Kurosawa ${ }^{1,3}$, Yasunori Nomura ${ }^{1}$, and T. Yanagida ${ }^{1,2}$ \\ ${ }^{1}$ Department of Physics, University of Tokyo, \\ Tokyo 113-0033, Japan \\ ${ }^{2}$ Research Center for the Early Universe, University of Tokyo, \\ Tokyo 113-0033, Japan \\ ${ }^{3}$ Yukawa Institute for Theoretical Physics, Kyoto University, \\ Kyoto 606-8201, Japan
}

\begin{abstract}
We discuss the anomalous $\mathrm{U}(1)$ gauge symmetry as a mechanism of generating the grand-unification scale. We conclude that unification to a simple group cannot be realized unless some parameters are "tuned", and that models with product gauge groups are preferred. We consider the " $R$-invariant natural unification" model with gauge groups $\mathrm{SU}(5)_{G U T} \times \mathrm{U}(3)_{H}$. In this model the doublet-triplet splitting problem is solved and the unwanted GUT relation $m_{s}=m_{\mu}$ is avoided maintaining $m_{b}=m_{\tau}$. Moreover, $R$-invariance suppresses the dangerous proton decays induced by dimension four and five operators.
\end{abstract}




\section{Introduction}

Three gauge coupling constants of the standard-model gauge interactions determined at the weak scale $\mu \simeq m_{Z}$ strongly suggest supersymmetric (SUSY) grand unification (GUT) of all gauge groups $\mathrm{SU}(3)_{C}, \mathrm{SU}(2)_{L}$ and $\mathrm{U}(1)_{Y}$ at a very high energy scale, $M_{G U T} \simeq 2 \times 10^{16} \mathrm{GeV}$. If the gravitational scale $M_{*} \simeq 2.4 \times 10^{18} \mathrm{GeV}$ is the fundamental cut-off scale of field-theory description of nature, it implies the presence of a mild hierarchy $M_{G U T} / M_{*} \simeq 10^{-2}$. It is very natural to consider such a hierarchy is a low-energy manifestation of more fundamental theory.

There is a mechanism which generates such a mild hierarchy. It is an anomalous $\mathrm{U}(1)_{A}$ gauge symmetry whose anomalies are cancelled by Green-Schwarz mechanism [1]. The anomalous $\mathrm{U}(1)_{A}$ gauge symmetry often appears in low energy effective theories of string theories [国]. Since the sum of $\mathrm{U}(1)_{A}$ charges is non-zero, $\operatorname{Tr} Q_{A} \neq 0$, in the anomalous $\mathrm{U}(1)_{A}$ gauge symmetry, Fayet-Iliopoulos (FI) term is induced at one-loop level [3]. In pertubative heterotic string theories it is given by

$$
\mathcal{L}_{\mathrm{FI}}=g_{A}^{2} \frac{\operatorname{Tr} Q_{A}}{192 \pi^{2}} M_{*}^{2} D_{A} \equiv \xi^{2} D_{A}
$$

If all fields have no VEVs, D-term is non-zero so that SUSY is broken. Instead, one usually assumes that some field $\phi$ has VEV to restore SUSY and define the normalization of $\mathrm{U}(1)_{A}$ charge so that $\phi$ has a $\mathrm{U}(1)_{A}$ charge -1 . In this normalization $\langle\phi\rangle=\xi$ and a mild hierarchy is generated as $\langle\phi\rangle / M_{*} \equiv \epsilon \sim \mathcal{O}\left(10^{-1}\right)$.

In this paper, we discuss the anomalous $\mathrm{U}(1)_{A}$ gauge symmetry as a mechanism of generating the mild hierarchy $M_{G U T} / M_{*}$. T We first show that the standard SUSY GUT based on a simple group cannot be realized unless some parameters are "tuned" and that models with product gauge groups are preferred instead. Here, we construct the " $R$-invariant natural unification" model [5] based on gauge groups $\mathrm{SU}(5)_{G U T} \times \mathrm{U}(3)_{H}$, in which the symmetry breaking scale $\simeq 2 \times 10^{16} \mathrm{GeV}$ is induced through the anomalous $\mathrm{U}(1)_{A}$ breaking. We also show that this model has various phenomenologically desirable features that the doublet-triplet splitting problem is solved, proton decays induced by dimension four and five operators [6, 7] are suppressed by $R$-symmetry and $m_{s} \neq m_{\mu}$

\footnotetext{
${ }^{1}$ There are several attempts to generate the hierarchy $M_{G U T} / M_{*}$ 四.
} 
without violating the GUT relation, $m_{b}=m_{\tau}$.

\section{Standard SUSY GUT models}

In this section, we show that it is difficult to generate the GUT scale through anomalous $\mathrm{U}(1)_{A}$ breaking in the standard SUSY SU(5) GUT models. In order to break SU(5) down to $\mathrm{SU}(3)_{C} \times \mathrm{SU}(2)_{L} \times \mathrm{U}(1)_{Y}$, a Higgs field $\Sigma$ in adjoint representation must have an appropriate vacuum expectation value (VEV), $\langle\Sigma\rangle / M_{*} \sim \mathcal{O}\left(10^{-2}\right)$. Since there is no scale but the breaking scale of the anomalous $\mathrm{U}(1)_{A}$ gauge symmetry, VEV of a field with a negative $\mathrm{U}(1)_{A}$ charge $-q$ is controlled by its $\mathrm{U}(1)_{A}$ charge and is of order $\epsilon^{q} M_{*}$ in general. That is, the field $\Sigma$ is required to have a negative $\mathrm{U}(1)_{A}$ charge -2 .

Now, we give a simple example for generating the GUT scale. Since $\Sigma$ has $\mathrm{U}(1)_{A}$ charge -2 , we have to introduce another field $\Sigma^{\prime}$ in adjoint representation with a positive $\mathrm{U}(1)_{A}$ charge 4 to have a nontrivial superpotential. Consider the following superpotential, ${ }^{2}$

$$
W=\kappa \Sigma^{\prime}\left\{\left(\frac{\phi}{M_{*}}\right)^{2} M_{*} \Sigma+\kappa^{\prime} \Sigma^{2}\right\}+\kappa^{\prime \prime}\left(\frac{\phi}{M_{*}}\right)^{8} M_{*} \Sigma^{\prime 2},
$$

where parameters, $\kappa$ 's, are assumed to be of order unity. There is a desirable SUSY vacuum which breaks $\mathrm{SU}(5)$ down to $\mathrm{SU}(3)_{C} \times \mathrm{SU}(2)_{L} \times \mathrm{U}(1)_{Y}$,

$$
\langle\Sigma\rangle / M_{*} \simeq\left(\epsilon^{2} / \kappa^{\prime}\right) \operatorname{diag}(2,2,2,-3,-3)
$$

The generated GUT scale is indeed $\mathcal{O}\left(\epsilon^{2} M_{*}\right)$ and the masses for broken gauge bosons, $M_{V}$, and those for $(\mathbf{8}, \mathbf{1}),(\mathbf{1}, \mathbf{3})$ components in $\Sigma$ and $\Sigma^{\prime}, M_{\Sigma}$, are of order $\epsilon^{2} M_{*}$, while masses for $\left(\mathbf{3}, \mathbf{2}^{\star}\right)$ and $\left(\mathbf{3}^{\star}, \mathbf{2}\right)$ components in $\Sigma^{\prime}, M_{X}$, of order $\epsilon^{8} M_{*} \ll M_{G U T}$. However, the disparity of the masses for various fields which belong to the same representation under SU(5) disturbs the unification of gauge coupling constants. We show it explicitly by considering the following combination of standard-model gauge coupling constants. From renormalization group equations (RGEs) at one-loop level, we get 8]

$$
\left(\frac{5}{\alpha_{1}}-\frac{3}{\alpha_{2}}-\frac{2}{\alpha_{3}}\right)\left(m_{Z}\right)=\frac{8}{2 \pi} \ln \left(\frac{m_{S U S Y}}{m_{Z}}\right)+\frac{12}{2 \pi} \ln \left(\frac{M_{V}^{2} M_{\Sigma}^{2}}{m_{Z}^{3} M_{X}}\right) .
$$

\footnotetext{
${ }^{2}$ The last term is necessary to give masses for $\left(\mathbf{3}, \mathbf{2}^{\star}\right)$ and $\left(\mathbf{3}^{\star}, \mathbf{2}\right)$ components in $\Sigma^{\prime}$, since $\left(\mathbf{3}, \mathbf{2}^{\star}\right)$ and $\left(\mathbf{3}^{\star}, \mathbf{2}\right)$ in $\Sigma$ are absorbed into broken gauge bosons.
} 
The observed values for standard-model gauge couplings constrain the combination of masses in the last term as

$$
\left(\frac{M_{V}^{2} M_{\Sigma}^{2}}{M_{X}}\right)^{1 / 3} \simeq M_{G U T} \simeq 2 \times 10^{16} \mathrm{GeV}
$$

On the other hand, $\left(M_{V}^{2} M_{\Sigma}^{2} / M_{X}\right)^{1 / 3}$ is almost equal to $M_{*}$ in the present model, since factors $\epsilon$ in $M_{V}, M_{\Sigma}$ and $M_{X}$ are cancelled out. It implies that the standard-model gauge coupling constants do not unify at the high-energy scale due to the mass splitting among various fields in the $\mathrm{SU}(5)$ multiplet $\Sigma^{\prime}$. We show, in Appendix, that such a situation cannot be avoided by adding fields in various representations. One possibility for avoiding it is to "tune" some parameters. For example, if we assume that $\kappa$ is not

of order unity but $\mathcal{O}\left(10^{-3}\right)$ in the superpotential Eq. (2), $M_{\Sigma} \simeq 10^{-3} \epsilon^{2} M_{*}$ and the combination $\left(M_{V}^{2} M_{\Sigma}^{2} / M_{X}\right)^{1 / 3}$ is $10^{-2} M_{*} \simeq 10^{16} \mathrm{GeV}$.

In this paper we pursue another possibility in which no "tuning" is required. It implies that we discard a "simple" idea that the standard-model gauge groups unify to one simple gauge symmetry. That is, we need GUT models with product gauge groups. As such models, we consider " $R$-invariant natural unification" model with a gauge group $\mathrm{SU}(5) \times \mathrm{U}(3)_{H}$ [5], since the anomalous $\mathrm{U}(1)_{A}$ gauge symmetry is naturally embedded in this model. Moreover, it has various desirable features in view of phenomenology.

\section{$3 \quad R$-invariant unification model}

Let us first discuss briefly the " $R$-invariant natural unification" model which is based on a SUSY $\mathrm{SU}(5)_{G U T} \times \mathrm{U}(3)_{H}$ gauge theory with an unbroken $R$ symmetry [5]. The $\mathrm{SU}(5)_{G U T}$ is the usual GUT gauge group and its coupling constant is in a perturbative regime, while the $\mathrm{U}(3)_{H}$ is a hypercolor gauge group whose coupling is strong at the GUT scale. Here, GUT means unification of all gauge groups, $\mathrm{SU}(3)_{C}, \mathrm{SU}(2)_{L}$ and $\mathrm{U}(1)_{Y}$, in the standard model. The quarks and leptons obey the usual transformation law under the GUT group $\mathrm{SU}(5)_{G U T}$ and they are all singlets of the hypercolor group $\mathrm{U}(3)_{H}$. A pair of Higgs multiplets $H_{r}$ and $\bar{H}^{r}(r=1, \cdots, 5)$ transform as $\mathbf{5}+\mathbf{5}^{\star}$ under the $\mathrm{SU}(5)_{G U T}$ and as singlets under the $\mathrm{U}(3)_{H}$.

All the matter multiplets introduced so far are the same as in the minimal SUSY 
$\mathrm{SU}(5)$ model. We now introduce six pairs of hyperquarks $Q_{\alpha}^{\rho}$ and $\bar{Q}_{\rho}^{\alpha}(\alpha=1, \cdots, 3$; $\rho=1, \cdots, 6)$ which transform as $\mathbf{3}$ and $\mathbf{3}^{\star}$ under the hypercolor $\mathrm{SU}(3)_{H}$ and have $\mathrm{U}(1)_{H}$ charges 1 and -1 , respectively $\left(\mathrm{SU}(3)_{H} \times \mathrm{U}(1)_{H} \equiv \mathrm{U}(3)_{H}\right)$. The first five pairs of $Q_{\alpha}^{r}$ and $\bar{Q}_{r}^{\alpha}(r=1, \cdots, 5)$ belong to $5^{\star}$ and 5 of $\mathrm{SU}(5)_{G U T}$ and the last pair of $Q_{\alpha}^{6}$ and $\bar{Q}_{6}^{\alpha}$ are singlets of $\mathrm{SU}(5)_{G U T}$. To cause a breaking of the total gauge group $\mathrm{SU}(5)_{G U T} \times \mathrm{U}(3)_{H}$ down to the standard-model gauge groups, $\mathrm{SU}(3)_{C} \times \mathrm{SU}(2)_{L} \times \mathrm{U}(1)_{Y}$, we introduce another chiral multiplet $X_{\beta}^{\alpha}$ coupled to $Q_{\alpha}^{r}$ and $\bar{Q}_{r}^{\alpha}$, which is an adjoint representation of the $\mathrm{SU}(3)_{H}$. Since $Q_{\alpha}^{r}$ and $\bar{Q}_{r}^{\alpha}$ are supposed to have VEV of order of the GUT scale, they must be trivial representations of the $R$ symmetry $\mathrm{U}(1)_{R}$ [5], and hence the $X_{\beta}^{\alpha}$ carry $R$-charge two. $R$ charges for all matter multiplets besides quark and lepton multiplets are given in Table 1.

To suppress unwanted nonrenormalizable interactions in superpotential we further impose an axial $\mathrm{U}(1)_{A}$ symmetry, under which the hyperquarks $Q_{\alpha}^{r}$ and $\bar{Q}_{r}^{\alpha}$ and the adjoint $X_{\beta}^{\alpha}$ transform as

$$
Q_{\alpha}^{r} \rightarrow e^{i \theta} Q_{\alpha}^{r}, \quad \bar{Q}_{r}^{\alpha} \rightarrow e^{i \theta} \bar{Q}_{r}^{\alpha}, \quad X_{\beta}^{\alpha} \rightarrow e^{-2 i \theta} X_{\beta}^{\alpha} .
$$

$\mathrm{U}(1)_{A}$ charges for all matter multiplets besides quark and lepton multiplets are also given in Table 1.

\begin{tabular}{|c|cc|cc|c|cc||cc|}
\hline & $Q_{\alpha}^{r}$ & $\bar{Q}_{r}^{\alpha}$ & $Q_{\alpha}^{6}$ & $\bar{Q}_{6}^{\alpha}$ & $X_{\beta}^{\alpha}$ & $H_{r}$ & $\bar{H}^{r}$ & $\phi$ & $\chi$ \\
\hline $\mathrm{U}(1)_{R}$ & 0 & 0 & 2 & 2 & 2 & 0 & 0 & 0 & 2 \\
$\mathrm{U}(1)_{A}$ & -2 & -2 & 2 & 2 & 4 & 0 & 0 & -1 & 4 \\
\hline
\end{tabular}

Table 1: Charge assignments in the Higgs sector.

Then, we have a superpotential $]^{5}$

$$
W=\lambda Q_{\alpha}^{r} \bar{Q}_{r}^{\beta} X_{\beta}^{\alpha}+h Q_{\alpha}^{r} \bar{Q}_{6}^{\alpha} H_{r}+\bar{h} Q_{\alpha}^{6} \bar{Q}_{r}^{\alpha} \bar{H}^{r} .
$$

Notice that the $R$ charges for $H_{r}$ and $\bar{H}^{r}$ are vanishing and those for $Q_{\alpha}^{6}$ and $\bar{Q}_{6}^{\alpha}$ are two. As shown in Ref. [5] we have the desirable vacua;

$$
\left\langle X_{\beta}^{\alpha}\right\rangle=\left\langle Q_{\alpha}^{6}\right\rangle=\left\langle\bar{Q}_{6}^{\alpha}\right\rangle=0,\left\langle H_{r}\right\rangle=\left\langle\bar{H}^{r}\right\rangle=0, \quad\left\langle Q_{\alpha}^{r}\right\rangle=v \delta_{\alpha}^{r}, \quad\left\langle\bar{Q}_{r}^{\alpha}\right\rangle=v \delta_{r}^{\alpha} .
$$

\footnotetext{
${ }^{3}$ The imposition of $\mathrm{U}(1)_{A}$ and $\mathrm{U}(1)_{R}$ alone allows nonrenormalizable interactions which include
} arbitrary powers of $H_{r} \bar{H}^{r} / M_{*}^{2}$. 
For $v \neq 0$, the total gauge group, $\mathrm{SU}(5)_{G U T} \times \mathrm{U}(3)_{H}$, is broken down to $\mathrm{SU}(3)_{C}$ $\times \mathrm{SU}(2)_{L} \times \mathrm{U}(1)_{Y}$ and hence the $v$ corresponds to the GUT scale. In these vacua, the color $\mathrm{SU}(3)_{C}$ is an unbroken linear combination of an $\mathrm{SU}(3)$ subgroup of the $\mathrm{SU}(5)_{G U T}$ and the hypercolor $\mathrm{SU}(3)_{H}$, and the hypercharge $\mathrm{U}(1)_{Y}$ is that of a $\mathrm{U}(1)$ subgroup of the $\mathrm{SU}(5)_{G U T}$ and the strong $\mathrm{U}(1)_{H}$. Thus, the gauge coupling constants $\alpha_{3}, \alpha_{2}$ and $\alpha_{1}$ of $\mathrm{SU}(3)_{C} \times \mathrm{SU}(2)_{L} \times \mathrm{U}(1)_{Y}$ are given by

$$
\alpha_{3} \simeq \frac{\alpha_{\mathrm{GUT}}}{1+\alpha_{\mathrm{GUT}} / \alpha_{3 H}}, \quad \alpha_{2}=\alpha_{\mathrm{GUT}}, \quad \alpha_{1} \simeq \frac{\alpha_{\mathrm{GUT}}}{1+\frac{1}{15} \alpha_{\mathrm{GUT}} / \alpha_{1 H}},
$$

where $\alpha_{3 H}$ and $\alpha_{1 H}$ are gauge coupling constants for the hypercolor $\mathrm{SU}(3)_{H}$ and $\mathrm{U}(1)_{H}$, respectively. We see that the unification of three gauge coupling constants, $\alpha_{3}, \alpha_{2}$ and $\alpha_{1}$, is practically achieved in a strong coupling regime of the hypercolor gauge interactions, that is, $\alpha_{3 H}$ and $\alpha_{1 H} \gg \mathcal{O}(1)$.

Interesting is that the color triplets $H_{a}$ and $\bar{H}^{a}(a=1, \cdots, 3)$ acquire masses of order $v$ together with the sixth hyperquarks $\bar{Q}_{6}^{\alpha}$ and $Q_{\alpha}^{6}$ in the vacua Eq. (8), while the weak doublets $H_{l}$ and $\bar{H}^{l}(l=4,5)$ remain massless since there are no partners for them to form $R$-invariant masses. The masslessness for these doublets is guaranteed by the unbroken $\mathrm{U}(1)_{R}$ symmetry.f

So far, the GUT scale $v$ is undetermined because of the presence of a flat direction in the present vacua. We identify the axial $\mathrm{U}(1)_{A}$ with the anomalous $\mathrm{U}(1)_{A}$ gauge symmetry and show that the GUT scale $v$ is determined by the breaking scale of the anomalous $\mathrm{U}(1)_{A}$. To obtain the desirable value for $v \simeq 2 \times 10^{16} \mathrm{GeV}$ we introduce a singlet $\chi$ with a $\mathrm{U}(1)_{A}$ charge +4 and the following superpotential

$$
W=k Q_{\alpha}^{r} \bar{Q}_{r}^{\alpha} \chi+\frac{k^{\prime}}{M_{*}^{2}} \phi^{4} \chi .
$$

Here, we have assumed $R$ charges for $\chi$ and $\phi$ are two and zero, respectively. Then, we get the GUT scale with a correct magnitude

$$
v \simeq \sqrt{\frac{k^{\prime}}{k}} \frac{\langle\phi\rangle^{2}}{M_{*}} \sim \mathcal{O}\left(10^{16} \mathrm{GeV}\right),
$$

for $k, k^{\prime} \sim \mathcal{O}(1)$.

\footnotetext{
${ }^{4}$ The $\mathrm{U}(1)_{R}$ symmetry is broken down to a discrete subgroup $Z_{4 R}$ by the hypercolor $\mathrm{SU}(3)_{H}$ anomaly. However, the unbroken subgroup $Z_{4 R}$ is sufficient to keep the Higgs doublets massless.
} 


\section{Quark and lepton mass matrices}

Let us turn to discuss quark and lepton mass matrices. It is very attractive to use the above $\phi$ field generating hierarchies in quark and lepton mass matrices [9, 10]. We tentatively assume $\mathrm{U}(1)_{A}$ charges for quark and lepton multiplets, $\mathbf{5}_{i}^{\star}$ and $\mathbf{1 0}_{i}(i=$ $1, \cdots, 3)$ as shown in Table 8 [11] where $\mathrm{U}(1)_{R}$ charges are also given. There are two cases for the assignment of $U(1)_{A}$ charges corresponding that $\tan \beta$ is small or large, where $\tan \beta$ is the ratio of the VEVs of the Higgs $(\tan \beta=\langle H\rangle /\langle\bar{H}\rangle)$. That is, $\tau=1$ for small $\tan \beta \sim \mathcal{O}(1)$ and $\tau=0$ for large $\tan \beta \sim \mathcal{O}(1 / \epsilon)$.

\begin{tabular}{|c|ccc|ccc||cc|}
\hline & $\mathbf{1 0}_{1}$ & $\mathbf{1 0}_{2}$ & $\mathbf{1 0}_{3}$ & $\mathbf{5}_{1}^{\star}$ & $\mathbf{5}_{2}^{\star}$ & $\mathbf{5}_{3}^{\star}$ & $H^{\prime}(\mathbf{4 5})$ & $H^{\prime}\left(\mathbf{4 5}^{\star}\right)$ \\
\hline $\mathrm{U}(1)_{R}$ & 1 & 1 & 1 & 1 & 1 & 1 & 0 & 2 \\
$\mathrm{U}(1)_{A}$ & 2 & 1 & 0 & $\tau+1$ & $\tau$ & $\tau$ & $-\tau-1$ & 4 \\
\hline
\end{tabular}

Table 2: Charge assignments for the matter fields.

Because of the $\mathrm{U}(1)_{A}$ invariance and $\langle\phi\rangle / M_{*}=\epsilon$, we obtain the Yukawa matrix for up-type quarks as

$$
\widehat{\lambda}_{u} \simeq\left(\begin{array}{ccc}
\epsilon^{4} & \epsilon^{3} & \epsilon^{2} \\
\epsilon^{3} & \epsilon^{2} & \epsilon \\
\epsilon^{2} & \epsilon & 1
\end{array}\right)
$$

and those for down-type quarks and charged leptons as

$$
\widehat{\lambda}_{d}=\widehat{\lambda}_{l} \simeq \epsilon^{\tau}\left(\begin{array}{ccc}
\epsilon^{3} & \epsilon^{2} & \epsilon^{2} \\
\epsilon^{2} & \epsilon & \epsilon \\
\epsilon & 1 & 1
\end{array}\right)
$$

Each element in the matrices has an undetermined coefficient of order unity. If $\epsilon \simeq 1 / 20$, the above mass matrices are roughly consistent with observations except for the unwanted GUT relations

$$
m_{s}=m_{\mu} \text { and } m_{d}=m_{e} .
$$

It is only the GUT condensation, $\left\langle Q_{\alpha}^{r}\right\rangle=v \delta_{\alpha}^{r}$ and $\left\langle\bar{Q}_{r}^{\alpha}\right\rangle=v \delta_{r}^{\alpha}$, that can break these unwanted GUT relations. In the above model, however, a possible operator is only

$$
\frac{1}{M_{*}^{2}} \mathbf{5}_{1}^{\star} \mathbf{1 0} \mathbf{H}_{1} \bar{H}\left\langle Q_{\alpha} \bar{Q}^{\alpha}\right\rangle
$$


for $\tau=1$ and nothing for $\tau=0$. Thus, it cannot lead to sufficient contributions to the quark and lepton mass matrices. A way to solve this problem is to introduce another pair of Higgs multiplets $H^{\prime}$ and $\bar{H}^{\prime}$ transforming as $\mathbf{4 5}$ and $\mathbf{4 5} 5^{\star}$ under the $\mathrm{SU}(5)_{\mathrm{GUT}}$. We assign $\mathrm{U}(1)_{A}$ charge for $\mathbf{4 5}$ so that the following Yukawa coupling is possible,

$$
5_{2}^{\star} 10_{2} H^{\prime}(45) .
$$

On the other hand, since the existence of $\mathbf{5}_{3}^{\star} \mathbf{1 0}_{3} H^{\prime}(\mathbf{4 5})$ would break the successful GUT relation $m_{b}=m_{\tau}, \mathrm{U}(1)_{A}$ and $\mathrm{U}(1)_{R}$ charges for $H^{\prime}(\mathbf{4 5})$ are determined as in Table 2 .

Now, massless Higgs doublet $\bar{H}_{f}$ is a linear combination of doublets, $\bar{H}_{f}\left(\mathbf{5}^{\star}\right)$ in $\bar{H}\left(\mathbf{5}^{\star}\right)$ and $H_{f}^{\prime}(\mathbf{4 5})$ in $H^{\prime}(\mathbf{4 5})$. Mixing angle $\theta$ is determined by the following superpotential

$$
W=H^{\prime}(\mathbf{4 5}) \bar{H}^{\prime}(\mathbf{4 5}) \frac{\phi^{2}}{M_{*}}\left(\text { or } \frac{\phi^{3}}{M_{*}^{2}}\right)+\frac{1}{M_{*}} \bar{H}\left(\mathbf{5}^{\star}\right) \bar{H}^{\prime}\left(\mathbf{4 5} 5^{\star}\right)\left\langle Q_{\alpha} \bar{Q}^{\alpha}\right\rangle,
$$

for $\tau=1$ (or $\tau=0$ ). Here, we have taken $\mathrm{U}(1)_{A}$ and $\mathrm{U}(1)_{R}$ charges for $\bar{H}^{\prime}\left(\mathbf{4 5}^{\star}\right)$ as in Table 2. We get $\theta \simeq\langle\phi\rangle^{2} / M_{*}^{2}\left(\right.$ or $\left.\langle\phi\rangle / M_{*}\right)$ where $\theta$ is defined as

$$
\bar{H}_{f}=\bar{H}_{f}\left(\mathbf{5}^{\star}\right) \cos \theta+H_{f}^{\prime}(\mathbf{4 5}) \sin \theta .
$$

This gives

$$
\begin{aligned}
\left\langle H_{f}^{\prime}(\mathbf{4 5})\right\rangle & \simeq \epsilon^{2}(\text { or } \epsilon)\left\langle\bar{H}_{f}\right\rangle, \\
\left\langle\bar{H}_{f}\left(\mathbf{5}^{\star}\right)\right\rangle & \simeq\left\langle\bar{H}_{f}\right\rangle,
\end{aligned}
$$

which leads to a required magnitude of the violation of the unwanted GUT relations, $m_{s}=m_{\mu}$ and $m_{d}=m_{e}$, as follows:

$$
\delta \widehat{\lambda}_{d} \simeq(-2) \times \epsilon^{\tau}\left(\begin{array}{ccc}
\epsilon^{3} & \epsilon^{2} & \epsilon^{2} \\
\epsilon^{2} & \epsilon & \epsilon \\
\epsilon & 0 & 0
\end{array}\right), \quad \delta \widehat{\lambda}_{l} \simeq 3 \times \epsilon^{\tau}\left(\begin{array}{ccc}
\epsilon^{3} & \epsilon^{2} & \epsilon^{2} \\
\epsilon^{2} & \epsilon & \epsilon \\
\epsilon & 0 & 0
\end{array}\right) .
$$

Note that the contribution from the GUT condensation for down-type quarks has an opposite sign to that for charged leptons. It shows that we can get the relation $m_{s}<m_{\mu}$ when the signs of $(2,2)$ elements in $\widehat{\lambda}_{d}$ and $\delta \widehat{\lambda}_{d}$ are opposite. If the GUT condensation is not taken into account, Cabbibo angle is given by $\epsilon$, which is smaller than the observed value 0.22 . However, due to the cancellation in $(2,2)$ element in the mass matrix for down-type quarks, we can get the desirable value.

\footnotetext{
${ }^{5}$ Another way to solve the problem is to lower the cut-off scale, $M_{*}$, and change the $\mathrm{U}(1)_{A}$ charge assignments in Table 11 .
} 


\section{Anomaly cancellations}

In this section, we discuss the cancellation of the $\mathrm{U}(1)_{A}$ mixed anomalies, $C_{G}$, with the gauge groups $G\left(G=\mathrm{SU}(5)_{G U T}, \mathrm{SU}(3)_{H}\right.$ and $\left.\mathrm{U}(1)_{H}\right)$. In the above model, we obtain $C_{G}$ as

$$
\begin{array}{llc}
C_{\mathrm{SU}(5)_{G U T}} & = & 35-\frac{21}{2} \tau, \\
C_{\mathrm{SU}(3)_{H}} & =4 & 4 \\
C_{\mathrm{U}(1)_{H}} & = & -48 .
\end{array}
$$

We find that $\mathrm{U}(1)_{A}$ has the negative anomaly coefficient $C_{\mathrm{U}(1)_{H}}$, which may not be expected in usual heterotic string theories.? In the heterotic string case there is the universal relation to the mixed anomaly coefficients as follows:

$$
\frac{C_{G}}{k_{G}}=\text { const. }
$$

where $k_{G}$ is the Kac-Moody level, since only the dilaton field $S$ plays a role in anomaly cancellations or, in other words, there is only one antisymmetric tensor field, $B_{\mu \nu}$ (GreenSchwarz mechanism) [1].

However, we can consider the case where various moduli fields other than the dilaton cancel these anomalies ("generalized" Green-Schwarz mechanism). Indeed, such a situation is realized in Type I and Type IIB string theories with orientifold compactifications [12]. The anomalous U(1) gauge symmetries in these theories have been studied recently and it has been revealed that several anomalous $\mathrm{U}(1)$ gauge symmetries exist corresponding to the existence of several antisymmetric tensors in twisted sectors.

The "generalized" Green-Schwarz mechanism is illustrated as follows. In this mechanism there are many moduli fields $M_{k}$ which are coupled to field strength superfields as

$$
\mathcal{L}_{\text {gauge }}=\int d^{2} \theta\left(k_{G} S+\sum_{k} c_{G}^{k} M_{k}\right) W_{G}^{\alpha} W_{G \alpha}
$$

and these moduli fields transform under the $\mathrm{U}(1)_{A}$ transformation, $V_{A} \rightarrow V_{A}+\frac{i}{2}\left(\Lambda-\Lambda^{\dagger}\right)$, as

$$
M_{k} \rightarrow M_{k}+i \delta_{k} \Lambda
$$

\footnotetext{
${ }^{6}$ Apparently, it seems possible that we add $\mathrm{U}(1)_{H}$-charged fields with positive $\mathrm{U}(1)_{A}$ charges so that the anomaly coefficient $C_{\mathrm{U}(1)_{H}}$ becomes positive. In that case, however, the gauge coupling constant for $\mathrm{U}(1)_{H}$ blows up below $M_{*}$.
} 
where $c_{G}^{k}$ and $\delta_{k}$ are model-dependent constants. This gives the nonuniversal relation to the mixed anomaly coefficients

$$
C_{G}=4 \pi^{2} \sum_{k} c_{G}^{k} \delta_{k}
$$

It shows that the three anomalies in Eqs. (22) can be cancelled by the non-linear transformation of the moduli fields $M_{k}$.

The origin of FI term is also different from that in the case of perturbative heterotic string theories. FI term is not generated at one-loop level but is given by the VEVs of moduli fields $\left\langle M_{k}\right\rangle$ at tree level as

$$
\mathcal{L}_{F I} \simeq-\sum_{k} \delta_{k}\left\langle M_{k}\right\rangle M_{*}^{2} D_{A} \equiv \xi^{2} D_{A}
$$

Although the VEVs of the moduli fields $\left\langle M_{k}\right\rangle$ are undetermined in the present framework, it seems natural to consider that the resulting FI term is one order smaller than $M_{*}$, i.e. $\epsilon=\xi / M_{*} \sim 10^{-1}$. Then, the conclusions in the previous sections are not affected.

\section{Summary and Discussions}

We have found that the idea that the GUT scale is generated through the anomalous $\mathrm{U}(1)_{A}$ breaking is compatible with the " $R$-invariant natural unification" model with gauge groups $\mathrm{SU}(5)_{G U T} \times \mathrm{U}(3)_{H}$. The Higgs field which breaks the GUT group has an anomalous $\mathrm{U}(1)_{A}$ charge and the unwanted GUT relations, $m_{s}=m_{\mu}$ and $m_{d}=m_{e}$, can be avoided in a simple manner without violating the successful GUT relation, $m_{b}=m_{\tau}$. Also, the doublet-triplet splitting problem is solved naturally in the present model.

As for proton decay, the vacuum in this model preserves the $\mathrm{U}(1)_{R}$ invariance and $R$-parity is included in the $\mathrm{U}(1)_{R}$ symmetry. Therefore, the dimension four and five operators contributing to the proton decay [6, 7] are suppressed. Instead, the process induced by the dimension six operators is dominant.

It is a crucial difference from the usual GUT models that the $\mathrm{SU}(3)_{C}$ is an unbroken linear combination of an $\mathrm{SU}(3)$ subgroup of the $\mathrm{SU}(5)_{G U T}$ and the hypercolor $\mathrm{SU}(3)_{H}$. It predicts a smaller value of the strong coupling constant $\alpha_{3}$. The current experimental values of $\alpha_{3}$ [13] are consistent with the present model [14]. 
We conclude this paper with a comment on neutrino masses [15]. Since neutrino masses are written by the effective operators, $\left(5^{\star} H\right)^{2}$, they are independent of $\mathrm{U}(1)_{A}$ charges of right handed neutrinos and are given by, [11

$$
\widehat{m}_{\nu} \simeq \epsilon^{2 \tau}\left(\begin{array}{ccc}
\epsilon^{2} & \epsilon & \epsilon \\
\epsilon & 1 & 1 \\
\epsilon & 1 & 1
\end{array}\right) \frac{m_{t}^{2}}{M_{*}}
$$

This mass matrix implies the large angle between the second and third generations and either small or large angle between the first and second ones. However, the mass of tau neutrino is of order of $10^{-5} \mathrm{eV}$ for $\tan \beta \sim \mathcal{O}\left(\epsilon^{-1}\right)$ or $10^{-7} \mathrm{eV}$ for $\tan \beta \sim \mathcal{O}(1)$. It is smaller by several orders than the scale indicated by the atmospheric neutrino oscillation [16]. There are two ways to generate a desirable mass scale. One way is to introduce the $\mathrm{U}(1)_{B-L}$ breaking scale as well as the anomalous $\mathrm{U}(1)_{A}$ breaking scale. The other is to extend the assignment of $\mathrm{U}(1)_{A}$ charges given in Table 1 and Table 2. This can be done because the assignment of $\mathrm{U}(1)_{A}$ charges is not uniquely determined by the following conditions: $Q \bar{Q}$ has charge -4 , the mixed anomaly $\mathrm{U}(1)_{H^{-}} \mathrm{U}(1)_{A}^{2}$ vanishes, $H \bar{H}$ has charge 0 in order to use the Giudice-Masiero mechanism for $\mu$ and $B \mu$ terms [17], and the superpotential (7) is $\mathrm{U}(1)_{A}$ invariant. The $\mathrm{U}(1)_{A}$ charges for various fields are given in Table 3 .

\begin{tabular}{|c|cc|cc|c|cc||cc|}
\hline & $Q_{\alpha}^{r}$ & $\bar{Q}_{r}^{\alpha}$ & $Q_{\alpha}^{6}$ & $\bar{Q}_{6}^{\alpha}$ & $X_{\beta}^{\alpha}$ & $H_{r}$ & $\bar{H}^{r}$ & $\phi$ & $\chi$ \\
\hline $\mathrm{U}(1)_{A}$ & $-2-p$ & $-2+p$ & $2-5 p$ & $2+5 p$ & 4 & $-4 p$ & $4 p$ & -1 & 4 \\
\hline & $\mathbf{1 0}_{1}$ & $\mathbf{1 0}_{2}$ & $\mathbf{1 0}_{3}$ & $\mathbf{5}_{1}^{\star}$ & $\mathbf{5}_{2}^{\star}$ & $\mathbf{5}_{3}^{\star}$ & $H^{\prime}(\mathbf{4 5})$ & $H^{\prime}\left(\mathbf{4 5}^{\star}\right)$ \\
\hline $\mathrm{U}(1)_{A}$ & $2+2 p$ & $1+2 p$ & $2 p$ & $\tau+1-6 p$ & $\tau-6 p$ & $\tau-6 p$ & $-\tau-1+4 p$ & $4-4 p$ \\
\hline
\end{tabular}

Table 3: Assignment of $\mathrm{U}(1)_{A}$ charges. $p$ is an arbitrary parameter. Taking $p=0$ corresponds to the assignment given in Table 11 and 2 .

For this charge assignment we are to replace the coefficient $\epsilon^{2 \tau}$ with $\epsilon^{-20 p+2 \tau}$ in Eq. (28). If we take $p=1 / 4$ and $\tau=1$, we can get desirable tau neutrino mass, $m_{\nu_{\tau}} \simeq \epsilon^{-3} m_{t}^{2} / M_{*} \sim 0.1 \mathrm{eV}$. With this charge assignment, dimension four baryon-numberviolating operators are completely suppressed by an unbroken discrete gauge symmetry. It is also desirable that no other scales are required to understand the neutrino masses. 


\section{Acknowledgments}

Y.N. thanks the Japan Society for the Promotion of Science for financial support. This work is supported in part by the Grant-in-Aid, Priority Area "Supersymmetry and Unified Theory of Elementary Particles" (\#707).

\section{Appendix}

In this appendix, we show by using the RGEs that the unification to SU(5) cannot be realized without "tuning" if all scales are generated by the breaking of the anomalous $\mathrm{U}(1)_{A}$ gauge symmetry. We consider generic SU(5) GUT models in which there are matter fields $\Psi_{I}$ with $\mathrm{U}(1)_{A}$ charges $q_{I}$ in various representations $\boldsymbol{R}_{\boldsymbol{I}}$ of $\mathrm{SU}(5)$ in addition to quark and lepton multiplets, $\mathbf{5}^{\star}{ }_{i}$ and $\mathbf{1 0}_{i}$, and the Higgs multiplets, $H(\mathbf{5})$ and $\bar{H}\left(\mathbf{5}^{\star}\right)$. The adjoint Higgs $\Sigma$ and $\Sigma^{\prime}$ are included in $\Psi_{I}$. Owing to the breaking of GUT, the

field in a representation $\boldsymbol{R}_{\boldsymbol{I}}$ is decomposed into fields $\psi_{I, i}$ in representations $\boldsymbol{r}_{\boldsymbol{I}, \boldsymbol{i}}$ of $\mathrm{SU}(3)_{C} \times \mathrm{SU}(2)_{L} \times \mathrm{U}(1)_{Y}$, which have SUSY masses $M_{I, i}$.

From the RGEs at one-loop level, we get

$$
\begin{aligned}
\left(\begin{array}{c}
1 / \alpha_{3} \\
1 / \alpha_{2} \\
1 / \alpha_{1}
\end{array}\right)\left(m_{Z}\right)= & \left(\begin{array}{c}
1 \\
1 \\
1
\end{array}\right)\left\{\frac{1}{\alpha_{5}\left(M_{*}\right)}-\frac{3}{2 \pi} \ln \left(\frac{M_{*}}{m_{Z}}\right)\right\}-\frac{1}{2 \pi}\left(\begin{array}{c}
4 \\
25 / 6 \\
5 / 2
\end{array}\right) \ln \left(\frac{m_{S U S Y}}{m_{Z}}\right) \\
& +\frac{1}{2 \pi}\left(\begin{array}{c}
4 \\
6 \\
10
\end{array}\right) \ln \left(\frac{M_{V}}{m_{Z}}\right)-\frac{1}{2 \pi} \times 2\left(\begin{array}{c}
1 / 2 \\
0 \\
1 / 5
\end{array}\right) \ln \left(\frac{M_{H_{C}}}{m_{Z}}\right) \\
& -\frac{1}{2 \pi} \sum_{(I, i)}^{\prime}\left(\begin{array}{l}
T_{3}\left(\boldsymbol{r}_{\boldsymbol{I}, \boldsymbol{i}}\right) \\
T_{2}\left(\boldsymbol{r}_{\boldsymbol{I}, \boldsymbol{i}}\right) \\
T_{1}\left(\boldsymbol{r}_{\boldsymbol{I}, \boldsymbol{i}}\right)
\end{array}\right) \ln \left(\frac{M_{I, i}}{m_{Z}}\right)
\end{aligned}
$$

where $M_{V}$ is the mass of the broken gauge bosons and $M_{H_{C}}$ is that of the colored Higgs. $T_{n}(\boldsymbol{r})(n=1,2,3)$ is the half of the Dynkin index for representation $\boldsymbol{r}$. The primed sum in the last term means that Goldstone modes for $\mathrm{SU}(5)$ breaking, $\left(\mathbf{3}, \mathbf{2}^{\star}\right)_{-5 / 6}$ and $\left(\mathbf{3}^{\star}, \mathbf{2}\right)_{5 / 6}$, are not included. Now, we take the following linear combination of standard-model gauge coupling constants in order to drop the contributions from fields in fundamental representations or its complex conjugates, 5 and $\mathbf{5}^{\star}$, including the colored Higgs,

$$
\left(\frac{5}{\alpha_{1}}-\frac{3}{\alpha_{2}}-\frac{2}{\alpha_{3}}\right)\left(m_{Z}\right)=\frac{8}{2 \pi} \ln \left(\frac{m_{S U S Y}}{m_{Z}}\right)+\frac{24}{2 \pi} \ln \left(\frac{M_{V}}{m_{Z}}\right)
$$




$$
+\frac{1}{2 \pi} \sum_{(I, i)}^{\prime}\left\{2 T_{3}\left(\boldsymbol{r}_{\boldsymbol{I}, \boldsymbol{i}}\right)+3 T_{2}\left(\boldsymbol{r}_{\boldsymbol{I}, \boldsymbol{i}}\right)-5 T_{1}\left(\boldsymbol{r}_{\boldsymbol{I}, \boldsymbol{i}}\right)\right\} \ln \left(\frac{M_{I, i}}{m_{Z}}\right) .
$$

The sum in the last term can be rewritten as the sum of the contributions from various representations $\boldsymbol{r}$ of $\mathrm{SU}(3)_{C} \times \mathrm{SU}(2)_{L} \times \mathrm{U}(1)_{Y}$,

$$
\frac{1}{2 \pi} \sum_{\boldsymbol{r}}\left\{2 T_{3}(\boldsymbol{r})+3 T_{2}(\boldsymbol{r})-5 T_{1}(\boldsymbol{r})\right\} \ln \left[\prod_{\substack{(I, i) \\ \boldsymbol{r}_{\boldsymbol{I}, \boldsymbol{i}}=\boldsymbol{r}}}^{\prime}\left(\frac{M_{I, i}}{m_{Z}}\right)\right] .
$$

Recall that $M_{I, i}$ are eigenvalues for the mass matrices, $M^{\boldsymbol{r}}$, for representations $\boldsymbol{r}$ which are determined when a specific superpotential is given. Even if we do not specified a superpotential, however, we are able to see the structure of the mass matrices using their $\mathrm{U}(1)_{A}$ charges as follows,

$$
\begin{aligned}
\prod_{\substack{(I, i) \\
\boldsymbol{r}_{\boldsymbol{I}, \boldsymbol{i}}=\boldsymbol{r}}} M_{I, i} & =\operatorname{det} M_{(I, i),(\bar{J}, \bar{\jmath})}^{\boldsymbol{r}} \simeq \operatorname{det}\left[\epsilon^{q_{I}+q_{\bar{J}}} M_{*}\right] \\
& \simeq\left\{\prod_{\substack{(I, i) \\
\boldsymbol{r}_{\boldsymbol{I}, \boldsymbol{i}}=\boldsymbol{r}}} \epsilon^{q_{I}} M_{*}^{1 / 2}\right\}\left\{\begin{array}{c}
\prod_{\substack{(\bar{J}, \bar{\jmath}) \\
\boldsymbol{r}_{\overline{\boldsymbol{J}}, \overline{\boldsymbol{j}}}=\boldsymbol{r}^{\star}}} \epsilon^{q_{\bar{J}}} M_{*}^{1 / 2}
\end{array}\right\} .
\end{aligned}
$$

Note that this equation is not valid for $\boldsymbol{r}=\left(\mathbf{3}, \mathbf{2}^{\star}\right)_{-5 / 6}$ and $\left(\mathbf{3}^{\star}, \mathbf{2}\right)_{5 / 6}$, since the mass matrices for $\left(\mathbf{3}, \mathbf{2}^{\star}\right)_{-5 / 6}$ and $\left(\mathbf{3}^{\star}, \mathbf{2}\right)_{5 / 6}$ have a zero eigenvalue corresponding to the Goldstone mode. Nevertheless, the relation similar to Eq. (32) holds also for $\boldsymbol{r}=\left(\mathbf{3}, \mathbf{2}^{\star}\right)_{-5 / 6}$ including the mass of broken gauge boson, $M_{V}$.

Suppose that the SU(5) multiplet $\Psi_{X}$ includes the Goldstone modes and has a negative anomalous $\mathrm{U}(1)_{A}$ charge $q_{X}<0$. Then, the mass for the broken gauge bosons is given by

$$
M_{V} \simeq g_{5}\left\langle\Psi_{X}\right\rangle \simeq \epsilon^{-q_{X}} M_{*} .
$$

The second term in Eq. (30) is rewritten as

$$
\begin{aligned}
\frac{24}{2 \pi} \ln \left(\frac{M_{V}}{m_{Z}}\right) & \simeq(-6) \times \frac{(-4)}{2 \pi} \ln \left(\frac{\epsilon^{-q_{X}} M_{*}}{m_{Z}}\right) \\
& =(-6) \times \frac{4}{2 \pi} \ln \left(\frac{\epsilon^{q_{X}} M_{*}^{1 / 2}}{m_{Z}^{1 / 2}}\right)+\frac{12}{2 \pi} \ln \left(\frac{M_{*}}{m_{Z}}\right)^{3},
\end{aligned}
$$


and the first term in Eq. (35) can be combined with the mass matrices for $\boldsymbol{r}=\left(\mathbf{3}, \mathbf{2}^{\star}\right)_{-5 / 6}$ and $\left(\mathbf{3}^{\star}, \mathbf{2}\right)_{5 / 6}$, giving the relation similar to Eq. (32) as

$$
\left(\frac{\epsilon^{q_{X}} M_{*}^{1 / 2}}{m_{Z}^{1 / 2}}\right)+\prod_{\substack{(I, i) \\ \boldsymbol{r}_{\boldsymbol{I}, \boldsymbol{i}=\boldsymbol{r}}}}^{\prime} M_{I, i}=\left(\frac{\epsilon^{q_{X}} M_{*}^{1 / 2}}{m_{Z}^{1 / 2}}\right)+\operatorname{det}^{\prime} M_{(I, i),(\bar{J}, \bar{\jmath})}^{\boldsymbol{r}} \simeq \operatorname{det}\left[\epsilon^{q_{I}+q_{\bar{J}}} M_{*}\right]
$$

Using Eqs. (32, 36), the Eq. (30) is expressed as

$$
\begin{aligned}
\left(\frac{5}{\alpha_{1}}-\frac{3}{\alpha_{2}}\right. & \left.-\frac{2}{\alpha_{3}}\right)\left(m_{Z}\right)=\frac{8}{2 \pi} \ln \left(\frac{m_{S U S Y}}{m_{Z}}\right)+\frac{12}{2 \pi} \ln \left(\frac{M_{*}}{m_{Z}}\right)^{3} \\
& +\frac{2}{2 \pi} \sum_{I} \ln \left(\frac{\epsilon^{q_{I}} M_{*}^{1 / 2}}{m_{Z}^{1 / 2}}\right)\left[\sum_{i}\left\{2 T_{3}\left(\boldsymbol{r}_{\boldsymbol{I}, \boldsymbol{i}}\right)+3 T_{2}\left(\boldsymbol{r}_{\boldsymbol{I}, \boldsymbol{i}}\right)-5 T_{1}\left(\boldsymbol{r}_{\boldsymbol{I}, \boldsymbol{i}}\right)\right\}\right] .
\end{aligned}
$$

The last terms vanish, since $\sum_{i} T_{n}\left(\boldsymbol{r}_{\boldsymbol{I}, \boldsymbol{i}}\right)=T\left(\boldsymbol{r}_{\boldsymbol{I}}\right)$ for each $I$ and $\sum_{i}\left\{2 T_{3}\left(\boldsymbol{r}_{\boldsymbol{I}, \boldsymbol{i}}\right)+3 T_{2}\left(\boldsymbol{r}_{\boldsymbol{I}, \boldsymbol{i}}\right)-\right.$ $\left.5 T_{1}\left(\boldsymbol{r}_{\boldsymbol{I}, \boldsymbol{i}}\right)\right\}=0$. Thus, we find that factors $\epsilon$ are cancelled out, so that the mass scale appearing in the RGE is not $M_{G U T}$ which is indicated by the observation but $M_{*}$. It implies that the coupling unification cannot be realized even if we add matters in various representations.

Note that in the above argument we have assumed that all unknown coefficients are of order unity and there are no cancellations in diagonalizing the mass matrices. As is remarked in Section 2, if we allow "tuning" of order $10^{-3}$ the coupling unification can be realized.

\section{References}

[1] M. Green and J. Schwarz, Phys. Lett. B149 (1984) 117.

[2] E. Witten, Phys. Lett. B149 (1984) 351.

[3] M. Dine, N. Seiberg, and E. Witten, Nucl. Phys. B289 (1987) 589;

J.J. Atick, L.J. Dixon and A. Sen, Nucl. Phys. B292 (1987) 109;

M. Dine, I. Ichinose and N. Seiberg, Nucl. Phys. B293 (1987) 253.

[4] H. Goldberg, Phys. Lett. B400 (1997) 301;

H.-C. Cheng, Phys. Lett. B410 (1997) 45;

M. Graesser, Phys. Rev. D59 (1999) 035007; 
C. Kolda and N. Polonsky, Phys. Lett. B433 (1998) 323;

Z. Chacko, M.A. Luty, and E. Ponton, Phys. Rev. D59 (1999) 035004.

[5] Izawa K.-I. and T. Yanagida, Prog. Theor. Phys. 97 (1997) 913.

[6] S. Dimopoulos and H. Georgi, Nucl. Phys. B193 (1981) 150.

[7] N. Sakai and T. Yanagida, Nucl. Phys. B197 (1982) 533;

S. Weinberg, Phys. Rev. D26 (1982) 287.

[8] J. Hisano, H. Murayama, and T. Yanagida, Phys. Rev. Lett. 69 (1992) 1014; Nucl. Phys. B402 (1993) 46.

[9] C.D. Froggatt and H.B. Nielsen, Nucl. Phys. B147 (1979) 277.

[10] L. Ibáñez and G.G. Ross, Phys. Lett. B332 (1994) 100;

P. Binétruy and P. Ramond, Phys. Lett. B350 (1995) 49;

V. Jain and R. Shrock, Phys. Lett. B352 (1995) 83;

E. Dudas, S. Pokorski, and C.A. Savoy, Phys. Lett. B356 (1995) 45.

[11] J. Sato and T. Yanagida, Phys. Lett. B430 (1998) 127; hep-ph/9809307;

W. Buchmüller and T. Yanagida, Phys. Lett. B445 (1999) 399.

[12] G. Aldazabal, A. Font, L.E. Ibáñez, and G. Violero, Nucl. Phys. B536 (1998) 29;

L.E. Ibáñez, R. Rabadán, and A.M. Uranga, Nucl. Phys. B542 (1999) 112;

E. Poppitz, Nucl. Phys. B542 (1999) 31;

Z. Lalak, S. Lavignac, and H.P. Nilles, hep-th/9903160.

[13] Particle Data Group, Eur. Phys. J. C3 (1998) 1.

[14] N. Arkani-Hamed, H.-C. Cheng and T. Moroi, Phys. Lett. B387 (1996) 529.

[15] H. Dreiner, G.K. Leontaris, S. Lola, G.G. Ross, and C. Scheich, Nucl. Phys. B436 (1995) 461;

P. Binétruy, S. Lavignac, and P. Ramond, Nucl. Phys. B477 (1996) 353;

P. Binétruy, S. Lavignac, S. Petcov, and P. Ramond, Nucl. Phys. B496 (1997) 3;

N. Irges, S. Lavignac, and P. Ramond, Phys. Rev. D58 (1998) 035003.

[16] Super-Kamiokande Collaboration, Y. Fukuda et al., Phys. Rev. Lett. 81 (1998) 1562.

[17] G.F. Giudice and A. Masiero, Phys. Lett. B206 (1988) 480. 